\title{
Comparison of Sofia Legionella FIA and BinaxNOW® Legionella urinary antigen card in two national reference centers
}

\author{
L. Beraud ${ }^{1}$ - K. Gervasoni ${ }^{3}$ - A. M. Freydiere ${ }^{1}$ - G. Descours ${ }^{1,2}$ - A. G. Ranc ${ }^{1,2}$ •

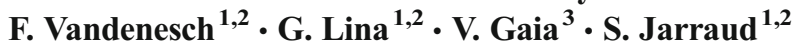

Received: 8 April 2015 / Accepted: 17 May 2015 / Published online: 20 June 2015

(C) The Author(s) 2015. This article is published with open access at Springerlink.com

\begin{abstract}
The Sofia Legionella Fluorescence Immunoassay (FIA; Quidel) is a recently introduced rapid immunochromatographic diagnostic test for Legionnaires' disease using immunofluorescence technology designed to enhance its sensitivity. The aim of this study was to evaluate its performance for the detection of urinary antigens for Legionella pneumophila serogroup 1 in two National Reference Centers for Legionella. The sensitivity and specificity of the Sofia Legionella FIA test were determined in concentrated and nonconcentrated urine samples, before and after boiling, in comparison with the BinaxNOW ${ }^{\circledR}$ Legionella Urinary Antigen Card (UAC; Alere). Compared with BinaxNOW ${ }^{\circledR}$ Legionella UAC, the sensitivity of the Sofia Legionella test was slightly higher in nonconcentrated urine samples and was identical in concentrated urine samples. The specificity of the Sofia Legionella FIA test was highly reduced by the concentration of urine samples. In nonconcentrated samples, a lack of specificity was observed in $2.3 \%$ of samples, all of them resolved by heat treatment. The Sofia Legionella FIA is a
\end{abstract}

The data were presented in part at the 8th International Conference on Legionella 2013 in Melbourne

S. Jarraud

sophie.jarraud@univ-lyon1.fr

1 French Reference Center for Legionella, Laboratory of Microbiology, Groupe Hospitalier Est, Hospices Civils de Lyon, Lyon, France

2 CIRI, International Center for Infectiology Research, Inserm, U1111 ; CNRS ; UMR5308, Université Lyon 1 ; École Normale Supérieure de Lyon, Lyon F-69008, France

3 Reference Center for Legionella, Laboratory of Microbiology, Department of Laboratory Medecine, Ente Ospedaliero Cantonale, Bellinzona, Switzerland sensitive test for detecting Legionella urinary antigens with no previous urine concentration. However, all positive samples have to be re-tested after boiling to reach a high specificity. The reading is automatized on the Sofia analyzer, which can be connected to laboratory information systems, facilitating accurate and rapid reporting of results.

\section{Introduction}

The accurate diagnosis of Legionella pneumonia is important for the treatment of patients by health care providers and for the control of Legionella epidemics by public health officials. Rapid urinary antigen detection kits such as lateral flow immunochromatographic assays detecting L. pneumophila serogroup 1 are the first-line diagnostic tests for Legionnaires' disease. In Europe, the $80 \%$ of cases were diagnosed using urinary antigen kits in 2009-2010 [1].

Various immunochromatographic membrane kits requiring no specialized laboratory equipment are commercially available. These tests are easy to perform and provide results in a few minutes, but they have not demonstrated optimal sensitivity [2-4]. Two approaches have been proposed to increase the sensitivity of the commercialized kits that are available: additional readings after a longer incubation time $[2,4-8]$ and the concentration of urine samples by centrifugation using filter units. Several studies showed an increase in the sensitivity of the BinaxNOW ${ }^{\circledR}$ Legionella Urinary Antigen Card (UAC) by using concentrated urine samples [4, 9-11].

The Sofia Legionella Fluorescence Immunoassay (FIA; Quidel, San Diego, CA, USA) is a recently introduced rapid diagnostic test for Legionnaires' disease (LD) that uses immunofluorescence technology coupled with an automatic reader to enhance its sensitivity. To date, no study has been published on the performance of this test compared with the 
BinaxNOW ${ }^{\circledR}$ Legionella UAC (Alere, Jouy-en-Josas, France), the kit that is most commonly used. The aim of this study was to evaluate the performance of Sofia Legionella FIA in comparison with BinaxNOW ${ }^{\circledR}$ Legionella UAC in concentrated and nonconcentrated urine samples at two National Reference Centers for Legionella.

\section{Materials and methods}

\section{Clinical specimens}

A total of 269 urine samples were tested in the French and Swiss National Reference Centers (centers 1 and 2 respectively: 199 in center 1 and 70 in center 2), corresponding to 179 prospective samples submitted for Legionella urinary antigen detection between January and March 2013 (150 in center 1 and 29 in center 2) and 90 repository urine samples collected from 2009 to 2013 from patients with known LD and stored at $-20{ }^{\circ} \mathrm{C}$ (49 in center 1 and 41 in center 2). Previously, urine samples had been classified as positive based on a BinaxNOW ${ }^{\circledR}$ Legionella UAC result obtained from concentrated urine samples.

A case of LD was defined according to the European Legionnaires' Disease Surveillance Network (ELDSNet) criteria [12], i.e., clinical and/or radiological evidence of pneumonia associated with positive urinary antigens and/or positive culture or PCR on respiratory samples. In our study, cases were diagnosed by positive urinary antigens using BinaxNOW ${ }^{\circledR}$ Legionella UAC on concentrated urine samples. Twenty-five cases were confirmed by culture on respiratory samples with Legionella pneumophila serogroup 1 strain and 7 of them were Mab3/1-negative.

\section{Legionella urinary antigen detection}

The BinaxNOW ${ }^{\circledR}$ Legionella $\mathrm{UAC}$ is a colorimetric immunochromatographic test. A sample was considered positive when both the control line and the test line were visible after $15 \mathrm{~min}$ of incubation at room temperature.

The Sofia Legionella FIA kit employs a lateral-flow immunofluorescence technique. The signal is only visible under UV light. The reading is performed after $10 \mathrm{~min}$ of incubation and interpreted using the SOFIA ${ }^{\mathrm{TM}}$ analyzer, which automatically scans the test strip, collects and analyzes the fluorescence data, and then calculates and reports the result in about $1 \mathrm{~min}$.

With the Binax Legionella Urinary Antigen EIA kit (Alere), a sample was considered positive when the mean absorbance value was three times the mean value of the negative control.

\section{Method}

The tests were performed according to the manufacturers' instructions. In addition, some samples were concentrated and heat-treated before testing. Urine samples yielding discrepant results before and after heat treatment were checked by using the Binax Legionella Urinary Antigen EIA kit.

Nonconcentrated urine samples were tested using Sofia Legionella FIA and the results were compared with those of nonconcentrated (centers 1 and 2) and those of concentrated urine samples (center 1 only) tested simultaneously using BinaxNOW ${ }^{\circledR}$ AUC.

Urinary concentration was performed in center 1 by centrifugation at 4,000 $\mathrm{g}$ for $10 \mathrm{~min}$ using Amikon Ultra-4 Ultracel$10 \mathrm{k}$ (Millipore Corporation, Bedford, MS, USA) [13].

In the two centers, urine samples yielding a positive result were retested after heating at $100{ }^{\circ} \mathrm{C}$ for $5 \mathrm{~min}$ and centrifugation for $15 \mathrm{~min}$ at $1,000 \mathrm{~g}$ to exclude false-positive results [14]. Any sample yielding a positive result after heating was considered true-positive. Any urine sample positive before heating and negative after heating was considered false-positive.

\section{Results}

Of the 269 nonconcentrated urine samples tested, the Sofia Legionella FIA test and the BinaxNOW ${ }^{\circledR}$ Legionella UAC yielded 172 and 181 negative results and 97 and 88 positive results respectively (Table 1). Of the 9 discrepant samples yielding a positive result with the Sofia Legionella FIA test, but a negative one with the BinaxNOW ${ }^{\circledR}$ Legionella UAC, 5 urine samples (4 in center 1 and 1 in center 2) yielded a negative result with the Sofia Legionella FIA test after heating. Four of them with a sufficient amount to be checked with the BinaxNOW ${ }^{\circledR}$ EIA showed a confirmed negative result. No respiratory samples were available for these 5 patients. The 4 other urine samples ( 2 in center 1 and 2 in center 2) remained positive with the Sofia Legionella FIA test after heating and yielded a positive result with the BinaxNOW ${ }^{\circledR}$ EIA before and after heating. For 2 of these patients, a Legionella $\mathrm{PCR}$ performed on respiratory samples was positive and in addition, for one of them a L. pneumophila serogroup 1-PCR was also positive. For the other one, the L. pneumophila serogroup 1-PCR was negative.

As several studies demonstrated that urine sample concentration increased the sensitivity of urinary tests, the results of the BinaxNOW ${ }^{\circledR}$ Legionella $\mathrm{UAC}$ on concentrated urine samples were compared with those of the Sofia Legionella FIA on nonconcentrated urine samples in center $1(n=199$; Table 2). The two reagents detected 49 positive samples, confirming the presence of Legionella urinary antigen. Among them, 2 samples had not been detected by the BinaxNOW ${ }^{\circledR}$ Legionella 
Table 1 Comparison of the results of the Sofia Legionella fluorescent immunoassay (FIA) and the BinaxNOW ${ }^{\circledR}$ Legionella urinary antigen card (UAC) using nonconcentrated urine samples $(n=269)$

\begin{tabular}{|c|c|c|c|c|c|c|}
\hline & & \multicolumn{5}{|c|}{ BinaxNOW ${ }^{\circledR}$ Legionella UAC nonconcentrated urine } \\
\hline & & \multicolumn{2}{|l|}{-} & \multicolumn{2}{|l|}{+} & \multirow[t]{2}{*}{ Total } \\
\hline & & Center 1 & Center 2 & Center 1 & Center 2 & \\
\hline Sofia Legionella FIA & - & 146 & 26 & 0 & 0 & 172 \\
\hline \multirow[t]{2}{*}{ Nonconcentrated urine } & + & $6^{\mathrm{a}}$ & $3^{\mathrm{a}}$ & 47 & 41 & 97 \\
\hline & Total & 181 & & 88 & & 269 \\
\hline
\end{tabular}

UAC before concentration. Four discrepant results were observed, yielding a positive result with the Sofia Legionella FIA and a negative one with the BinaxNOW ${ }^{\circledR}$ Legionella UAC. These samples were confirmed as negative by the Sofia Legionella FIA after heating, corresponding to false-positive results as previously described in Table 1 .

Although the manufacturer of the Sofia Legionella FIA does not recommend urine concentration, we evaluated its impact on 199 concentrated urine samples in center 1. Eleven urine samples were detected as being positive with use of the Sofia Legionella FIA, but negative with use of the BinaxNOW ${ }^{\circledR}$ Legionella UAC. Among these samples, 9 turned out to have negative results after heating, confirming false positivity. In 1 case there was not enough of the urine sample available for retesting after heating; thus, it was classified as invalid. The last sample remained positive after heating, but was detected as being negative by the BinaxNOW ${ }^{\circledR}$ EIA. Legionella spp. and $L$. pneumophila PCR performed on respiratory samples were negative for this patient.

\section{Discussion}

In this work, we evaluated the Sofia Legionella FIA, a recently introduced rapid diagnostic test for the detection of L. pneumophila serogroup 1 urinary antigens, which uses immunofluorescence technology to enhance its sensitivity.

Although the manufacturers do not recommend urine concentration, the centrifugal ultrafiltration method for rapid concentration of $L$. pneumophila urinary antigen was evaluated. The Legionella urinary antigen is stable at $100{ }^{\circ} \mathrm{C}$ for $30 \mathrm{~min}$ [15]; therefore, all positive samples were retested as described previously after boiling to suppress nonspecific reactions and confirm positive results $[9,14,16,17]$.

Our results demonstrate that the Sofia Legionella FIA test exhibits higher sensitivity than the BinaxNOW ${ }^{\circledR}$ Legionella UAC, since it allowed the detection of all the LD-positive patients with nonconcentrated urine samples. This study also confirms that the sensitivity of BinaxNOW ${ }^{\circledR}$ Legionella UAC is enhanced by the concentration of urine without any decrease in specificity. As a matter of fact, 2 nonconcentrated urine samples that tested negative with the BinaxNOW ${ }^{\circledR}$ Legionella UAC yielded a positive result after concentration. Thus, these two tests demonstrated similar performance in terms of sensitivity only if urine samples are concentrated for the BinaxNOW ${ }^{\circledR}$ Legionella UAC.

The major limitation of the Sofia Legionella FIA is the lower specificity compared with the BinaxNOW ${ }^{\circledR}$ Legionella UAC that was detected in both concentrated and nonconcentrated urine samples. As a matter of fact, Quidel recommends using the Sofia Legionella FIA in nonconcentrated urine samples. Our results confirm this important point, since the number of false-positive results increases from 4 before to 11 after concentration. Moreover, 1 out of the 11 false-positive results in concentrated urine samples remained positive after heating.

These results of sensitivity and specificity were further confirmed in 214 new prospective urine samples tested
Table 2 Comparison of the results of the Sofia Legionella fluorescent immunoassay (FIA) using nonconcentrated urine samples and the BinaxNOW ${ }^{\circledR}$ Legionella urinary antigen card (UAC) using concentrated urine samples in center $1(n=199)$

\begin{tabular}{llrrr}
\hline & & \multicolumn{2}{l}{ BinaxNOW ${ }^{\circledR}$ Legionella UAC concentrated urine } \\
\cline { 3 - 5 } & & \multicolumn{1}{c}{} & + & Total \\
\hline Sofia Legionella FIA & - & 146 & 0 & 146 \\
Nonconcentrated urine & + & $4^{\mathrm{a}}$ & 49 & 53 \\
& Total & 150 & 49 & 199 \\
\hline
\end{tabular}

${ }^{a}$ These 4 urine samples yielded a negative result after heating and corresponded to the same samples as in the cells marked $^{\text {a }}$ in Table 1 (false-positive results) 
between March and June 2014 in center 1 with Sofia Legionella FIA performed on nonconcentrated samples and the BinaxNOW ${ }^{\circledR}$ Legionella UAC on concentrated samples. Three positive urine samples were equally detected by the two kits and boiling urine samples eliminated 6 false-positive results with use of the Sofia Legionella FIA.

Finally, without heat treatment, a lack of specificity was observed for $2.3 \%$ of all samples tested in this study (11 out of 483). Because of the consequences of false-positive results on the patient outcome and on outbreak investigation $[17,18]$, the recommendation of boiling any positive urine to differentiate a false-positive result from a true one should be recommended in the manufacturer's instructions.

The Sofia Legionella FIA may represent a next step in the evolution of immunoassays, combining lateral flow and fluorescent antibody detection formats. The choice of fluorescent rather than colorimetric detection is at least partially responsible for the improved sensitivity demonstrated by the Sofia Legionella FIA, and this improvement has also been demonstrated for Influenza virus detection in comparison with several colorimetric lateral flow devices [19-22]. Because of its automated reading rather than subjective readings of the colorimetric bands, this assay produces objective data. Moreover, the Sofia analyzer can be connected to laboratory information systems to facilitate the accurate and rapid reporting of results.

Acknowledgements The authors kindly thank the two technicians of our laboratory, Karine Droitcourt and Marielle Siffert, for performing antigen urinary assays.

Conflict of interest The authors declare that they have no conflict of interest.

Funding Sofia Legionella FIA kits and equipment were kindly provided by Quidel Corp. Hospices Civils de Lyon received a grant from Quidel to support the evaluation of the Sofia Legionella FIA kits.

Open Access This article is distributed under the terms of the Creative Commons Attribution 4.0 International License (http:// creativecommons.org/licenses/by/4.0/), which permits unrestricted use, distribution, and reproduction in any medium, provided you give appropriate credit to the original author(s) and the source, provide a link to the Creative Commons license, and indicate if changes were made.

\section{References}

1. Beauté J, Zucs P, de Jong B, European Legionnaires' Disease Surveillance Network (2013) Legionnaires disease in Europe, 2009-2010. Euro Surveill 18:20417

2. Bruin JP, Diederen BM (2012) Evaluation of Meridian TRU Legionella $^{\circledR}$, a new rapid test for detection of Legionella pneumophila serogroup 1 antigen in urine samples. Eur J Clin Microbiol Infect Dis 32:333-334

3. Svarrer CW, Lück C, Elverdal PL, Uldum SA (2012) Immunochromatic kits Xpect Legionella and BinaxNOW Legionella for detection of Legionella pneumophila urinary antigen have low sensitivities for the diagnosis of Legionnaires' disease. J Med Microbiol 61:213-217

4. Yzerman EP, den Boer JW, Lettinga KD, Schellekens J, Dankert J, Peeters M (2002) Sensitivity of three urinary antigen tests associated with clinical severity in a large outbreak of Legionnaires' disease in The Netherlands. J Clin Microbiol 40:3232-3236

5. Diederen BM, Bruin JP, Scopes E, Peeters MF, IJzerman EP (2009) Evaluation of the Oxoid Xpect Legionella test kit for detection of Legionella pneumophila serogroup 1 antigen in urine. J Clin Microbiol 47:2272-2274

6. Bruin JP, Peeters MF, Ijzerman EP, Diederen BM (2010) Evaluation of Legionella V-TesT for the detection of Legionella pneumophila antigen in urine samples. Eur J Clin Microbiol Infect Dis 29:899-900

7. Held J (2012) Increasing the sensitivity of the BinaxNOW Legionella urinary antigen immunochromatographic test by additional readings at later time points. J Med Microbiol 61:884-885

8. Diederen BM, Peeters MF (2007) Evaluation of the SAS Legionella Test, a new immunochromatographic assay for the detection of Legionella pneumophila serogroup 1 antigen in urine. Clin Microbiol Infect 13:86-88

9. Domínguez JA, Galí N, Pedroso P, Fargas A, Padilla E, Manterola JM, Matas L (1998) Comparison of the Binax Legionella urinary antigen enzyme immunoassay (EIA) with the Biotest Legionella Urin antigen EIA for detection of Legionella antigen in both concentrated and nonconcentrated urine samples. J Clin Microbiol 36:2718-2722

10. Guerrero C, Toldos CM, Yagüe G, Ramírez C, Rodríguez T, Segovia M (2004) Comparison of diagnostic sensitivities of three assays (Bartels enzyme immunoassay [EIA], Biotest EIA, and Binax NOW immunochromatographic test) for detection of Legionella pneumophila serogroup 1 antigen in urine. J Clin Microbiol 42:467-468

11. Domínguez JA, Manterola JM, Blavia R, Sopena N, Belda FJ, Padilla E, Giménez M, Sabrià M, Morera J, Ausina V (1996) Detection of Legionella pneumophila serogroup 1 antigen in nonconcentrated urine and urine concentrated by selective ultrafiltration. J Clin Microbiol 34:2334-2336

12. http://ecdc.europa.eu (ECDC Portal $>$ English $>$ Activities $>$ Surveillance $>$ European Surveillance Networks/ECDC Disease Specific Surveillance $>$ European Legionnaires' Disease Surveillance Network (ELDSNet) $>$ EU case definition). Accessed 19 February 2015

13. Blanco S, Prat C, Pallarés MA, Matas L, Domínguez J (2004) Centrifugal ultrafiltration method for rapid concentration of Legionella pneumophila urinary antigen. J Clin Microbiol 42:4410

14. Doskeland SO, Berdal BP (1980) Bacterial antigen detection in body fluids: methods for rapid antigen concentration and reduction of nonspecific reactions. J Clin Microbiol 11:380-384

15. Kohler RB, Zimmerman SE, Wilson E, Allen SD, Edelstein PH, Wheat LJ, White A (1981) Rapid radioimmunoassay diagnosis of Legionnaires' disease: detection and partial characterization of urinary antigen. Ann Intern Med 94:601-605

16. Birtles RJ, Harrison TG, Samuel D, Taylor AG (1990) Evaluation of urinary antigen ELISA for diagnosing Legionella pneumophila serogroup 1 infection. J Clin Pathol 43:685-690

17. Pontoizeau C, Dangers L, Jarlier V, Luyt CE, Guiller E, Fievet MH, Lecsö-Bornet M, Aubry A, Brossier F (2014) Ruling out falsepositive urinary Legionella pneumophila serogroup 1 and Streptococcus pneumoniae antigen test results by heating urine. J Clin Microbiol 52:4347-4349

18. Rota MC, Fontana S, Montaño-Remacha C, Scaturro M, Caporali MG, Vullo V, Scorzolini L, Ercole A, Ricci ML (2014) Legionnaires' disease pseudoepidemic due to falsely positive urine antigen test results. J Clin Microbiol 52:2279-2280

19. Rath B, Tief F, Obermeier P, Tuerk E, Karsch K, Muehlhans S, Adamou E, Duwe S, Schweiger B (2012) Early detection of influenza 
$\mathrm{A}$ and $\mathrm{B}$ infection in infants and children using conventional and fluorescence-based rapid testing. J Clin Virol 55:329-333

20. Lewandrowski K, Tamerius J, Menegus M, Olivo PD, Lollar R, Lee-Lewandrowski E (2013) Detection of influenza A and B viruses with the Sofia analyzer: a novel, rapid immunofluorescencebased in vitro diagnostic device. Am J Clin Pathol 139:684-689
21. Leonardi GP, Wilson AM, Zuretti AR (2013) Comparison of conventional lateral-flow assays and a new fluorescent immunoassay to detect influenza viruses. J Virol Methods 189:379-382

22. Lee CK, Cho CH, Woo MK, Nyeck AE, Lim CS, Kim WJ (2012) Evaluation of Sofia fluorescent immunoassay analyzer for influenza A/B virus. J Clin Virol 55:239-243 